\title{
Development and study of hybrid organic-colloidal quantum dot systems
}

\author{
Kostantinos Bourdakos*, D. M. Nanditha Dissanayake, Richard J. Curry. \\ Advanced Technology Institute, University of Surrey, Guildford, Surrey, GU2 7XH. UK.
}

\begin{abstract}
Abstract here

Keywords: Fullerene, quantum dot, $\mathrm{PbS}$

\section{INTRODUCTION}

Since the demonstration of a rapid synthesis method for the preparation of colloidal quantum dots ${ }^{1,2.3}$ (QDs) interest in their application to a wide range of optoelectronic devices has been strong. A wide range of material systems have been studied that include $\mathrm{CdSe}^{4}, \mathrm{CdS}^{4}, \mathrm{InAs}^{5}, \mathrm{PbS}^{6}$ and $\mathrm{PbSe}^{7}$ amongst others with direct optical bandgaps ranging from the visible to near infrared (NIR) respectively. The significant interest in such QDs is related to the fact that the properties of these systems are comparable to the ideal quantum mechanic "particle in a box" with a discrete density of states that has also lead to them being referred to as 'artificial atoms'. The origin of these properties are directly related to the diameter of the QDs. In such systems when the diameter of the QD is below that of the bulk excitonic Bohr radius strong confinement of the charges within occurs and thus by varying the size of the QD one can control the energy of the electronic states ${ }^{2,8,9}$.
\end{abstract}

The ability to directly control the optical bandgap and hence the absorption and emission properties of these materials has been used to demonstrate a number of devices including LEDs $^{10}$, photovoltaic and solar cells ${ }^{11,12}$, lasers and optical gain media ${ }^{13,14}$, photonics ${ }^{15,16}$, biological fluorescence labelling ${ }^{17}$ and as components in molecular electronics ${ }^{18}$. Of these the use of QDs as optically active components is particularly attractive in organic light emitting diodes (OLEDs) and organic photovoltaic devices (OPVs). The use of QDs in OLEDs ${ }^{19,20}$ enables emission to obtained at NIR wavelengths that are much lower in energy than is possible using organic molecules alone relying on $\pi-\pi^{*}, \mathrm{n}-\pi^{*}$ or metal to ligand charge transfer processes. Additionally, in comparison with the intra-atomic $4 f-4 f$ transitions of rare-earth ions in organolanthanide complexes ${ }^{21}$ the emission at these NIR wavelengths (e.g. $\sim 1.53 \mu \mathrm{m}$ ) in QDs is highly efficient in nature. Despite this fact the efficiency of OLEDs that incorporate QDs are well below those of conventional OLED devices emitting in the visible region of the spectrum and significant further work is required on these hybrid organic-QD systems in order to fully understand and optimize them.

The use of organic-QD hybrid systems in OPV devices is also an area of some intense study due to the ability to tune the optical response of solar cells for example. Current organic devices are limited to absorbing light at energy above $~ 1.5$ $\mathrm{eV}(\sim 850 \mathrm{~nm})$ again due to the nature of the $\pi-\pi^{*}$ optical states. As a result a significant proportion of the suns energy cannot be utilized in traditional OPV devices. The incorporation of NIR absorbing QDs into such devices would therefore enable this untapped energy to be accessed. To date however the majority of OPV devices incorporating QDs have used Cd-based QDs (e.g. CdS, CdSe, CdTe) and have therefore not utilized longer NIR wavelengths. A study by McDonald $e t a^{22}$. and Maria et al. ${ }^{23}$ have demonstrated the use of PbS QDs in conjunction with the polymers poly[2methoxy-5-(2-ethylhexyloxy)-1,4-phenylenevinylene] (MEH-PPV) and poly(3-octlythiophene) P3OT in devices demonstrating NIR sensitization.

In addition to the ability to obtain NIR sensitization in OPVs the use of PbS and PbSe QDs may also allow the thermodynamic limit for quantum efficiency of $31 \%$ for a single junction to be overcome. Work by Nozik has shown that due to the effective mass of the electrons and holes in these QDs being similar a process of impact ionization (inverse Auger) becomes competitive with other relaxation mechanisms for highly excited electron-hole pairs within the $\mathrm{QD}^{24}$. 
This has since been confirmed experimentally ${ }^{25}$, though not in a working OPV device, to occur for excitation energy above 2.2 times the QD bandgap in the PbSe system ${ }^{26}$.

When considering the development of hybrid organic-QD systems for such applications it is important to understand the interaction of the constituent species and how they affect the optical and electronic properties of devices utilizing them. With this in mind we have undertaken a study of hybrid fullerene-QD systems and in particular $\mathrm{C}_{60}-\mathrm{PbS}$ and [6,6]Phenyl-C61 butyric acid methyl ester (PCBM)-PbS. The fullerene molecules chosen for this study are of particular interest in the context of OPV devices due to their high electron mobility in comparison to other small molecule organics often used in this role. In this paper we report initial results based upon studies of the optical properties of composite samples prepared as described below. These results have significant implications for the future development of small molecule hybrid organic-QD systems.

\section{EXPERIEMENTAL}

The PbS QD used in this study were obtained from Evident Technologies. $\mathrm{C}_{60}$ (99.99\%) and PCBM (99.5\%) were purchased from American Dye Source and Solenne respectively and used without further purification. Poly(methymethacrylate) (PMMA $\mathrm{M}_{\mathrm{w}}=120,000$ ) was obtained from Sigma-Aldrich. All solvents used were of reagent grade. Stock solutions of PbS $(2.5 \mathrm{mg} / \mathrm{ml}), \mathrm{C}_{60}(2.5 \mathrm{mg} / \mathrm{ml})$, PCBM $(2.5 \mathrm{mg} / \mathrm{ml})$ and PMMA dissolved in toluene were prepared of known concentration. Samples were then prepared through combining each solution in the desired concentration from the stock solutions providing a number samples with varying $\mathrm{PbS}$ :Fullerene concentration ratios in PMMA hosts (Table 1). These were then drop cast onto cleaned quartz substrates and the toluene allowed to evaporate resulting in thin film samples.

Table 1. Description of samples studied.

\begin{tabular}{lc}
\hline \multicolumn{1}{c}{ Sample constituents } & PbS: Fullerene Weight Ratio \\
\hline PMMA & \\
PMMA + $\mathrm{C}_{60}$ & $0: 1$ \\
PMMA + PbS & $1: 0$ \\
PMMA + PbS + $\mathrm{C}_{60}$ & $9: 1$ \\
PMMA + PbS + $\mathrm{C}_{60}$ & $4.5: 1$ \\
PMMA + PbS + 60 & $1: 1$ \\
PMMA + PbS + $\mathrm{C}_{60}$ & $1: 4.5$ \\
PMMA + PbS + 60 & $1: 9$ \\
PMMA + PCBM & $0: 1$ \\
PMMA + PbS + PCBM & $9: 1$ \\
PMMA + PbS + PCBM & $4.5: 1$ \\
PMMA + PbS + PCBM & $1: 1$ \\
PMMA + PbS + PCBM & $1: 4.5$ \\
PMMA + PbS + PCBM & $1: 9$ \\
\hline
\end{tabular}

Absorption spectra were obtained using a Varian Cary 5000 spectrophotometer. Photoluminescence measurements were obtained using various excitation wavelengths as described below using modulated Ar-ion and diode laser sources. The emission was dispersed in a Bentham TmC300 spectrometer and collected using Si and InGaAs photodiodes. The detected signal was measured using a Signal Recovery 7265 lock-in amplifier. All spectra are corrected for the system response.

\section{RESULTS}

\section{Absorption}



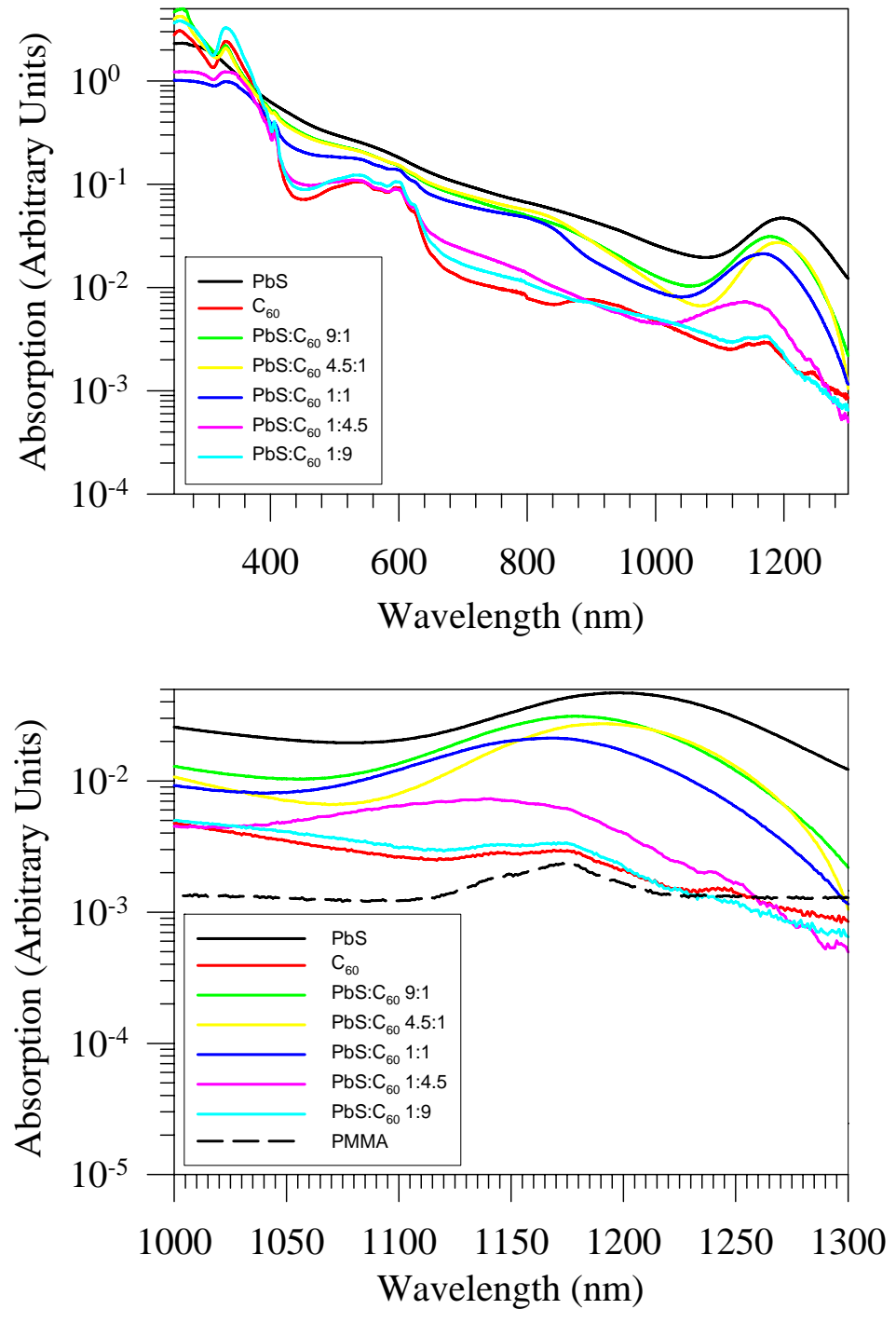

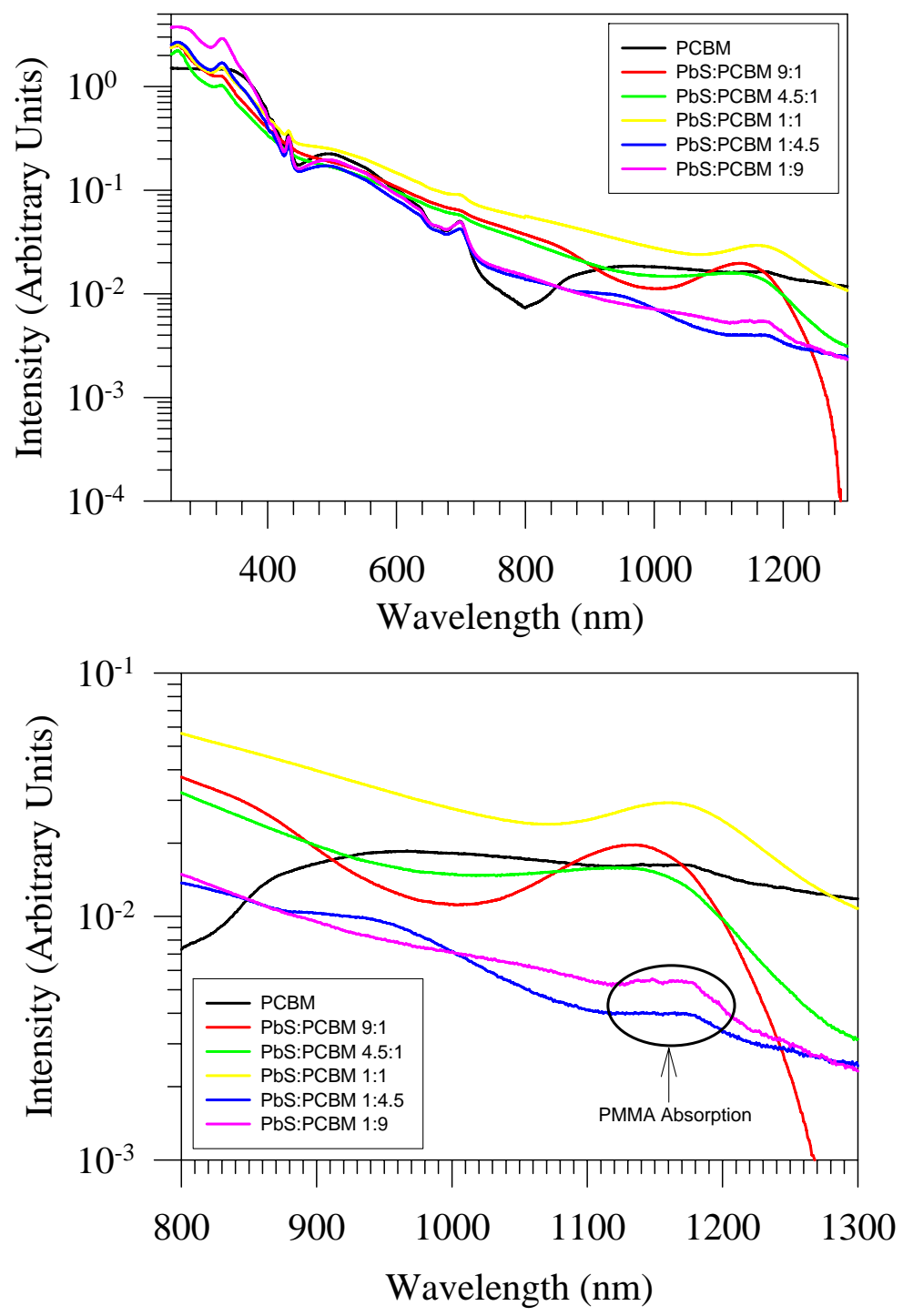

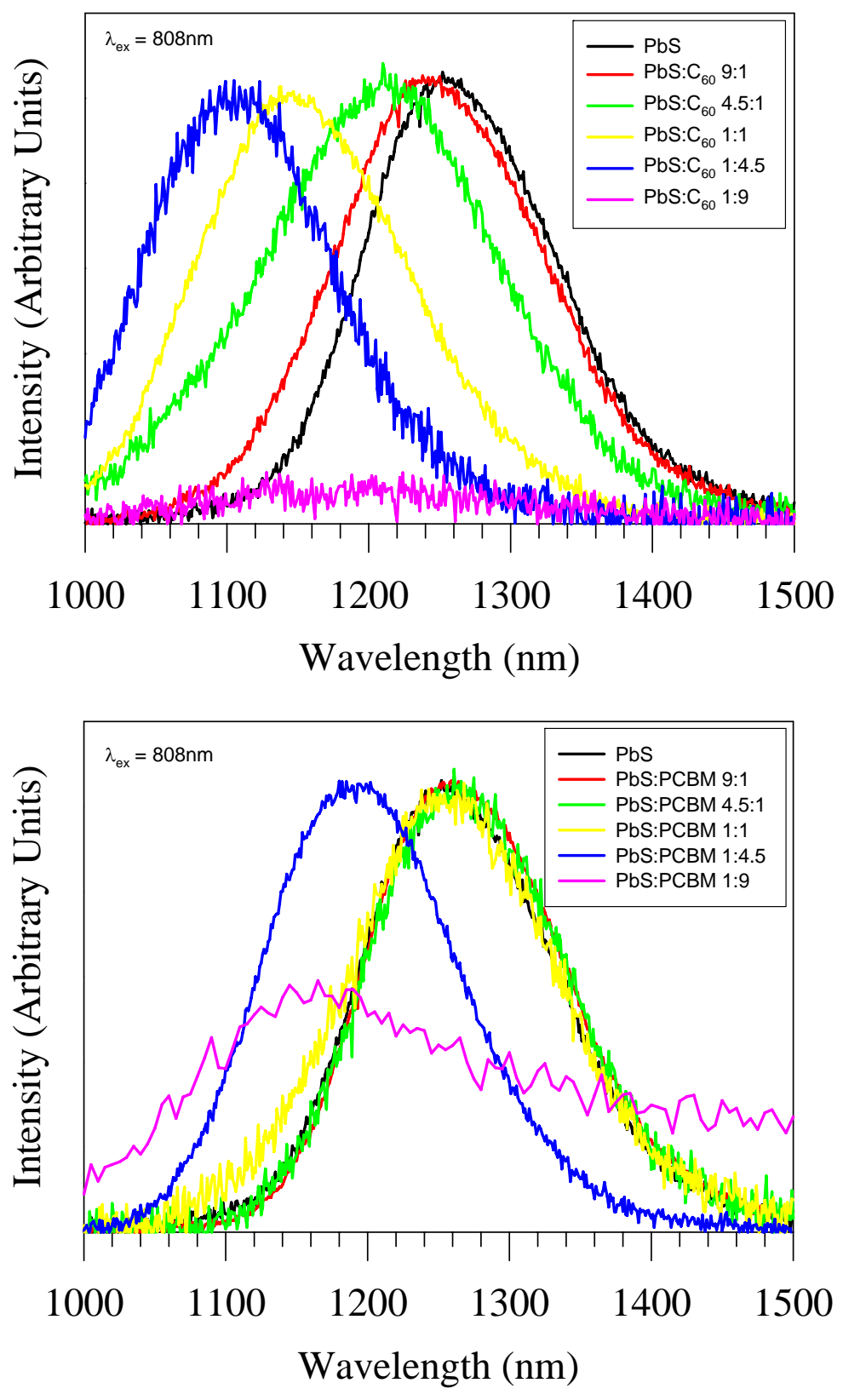

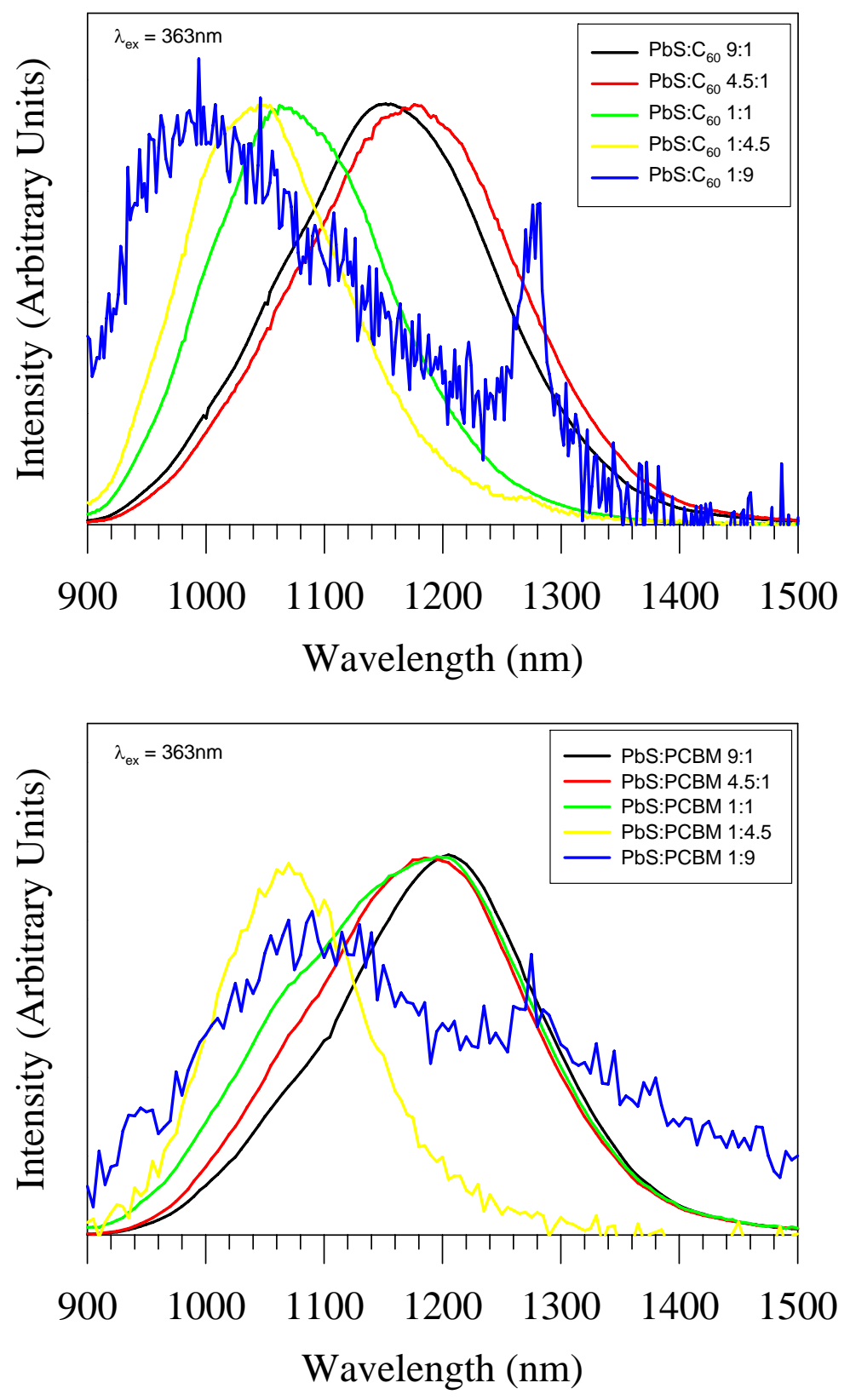


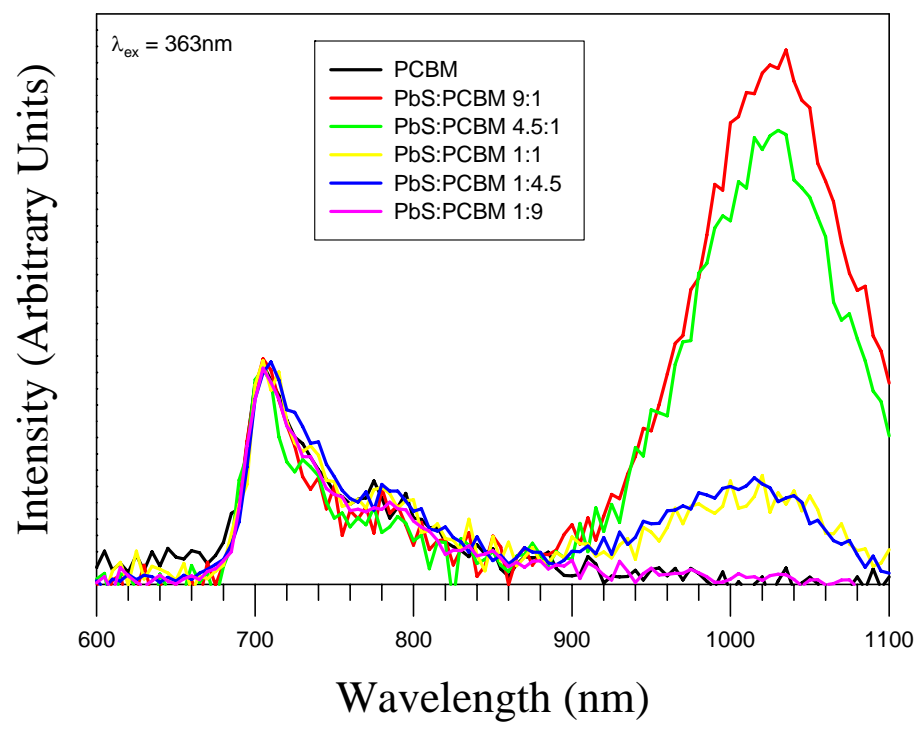

1. C.B. Murrey, D.B. Norris and M.G. Bawendi, J. Am. Chem. Soc., 115, 8706 (1993)

2. A.P. Alivisatos. Science, 271, 933-937 (1996).

3. N.C. Greenham, X. Peng, A.P. Alivisatos, Phys. Rev. B., 54, 17628 (1996).

4. T. Vossmeyer, L. Kasikas, M. Giersig, I.G. Popovic, K. Diesner, A. Chemseddine, A. Eychmuller and H. Weller, J. Phys. Chem. 98, 7665 (1994).

5. A.A. Guzelian, U. Banin, A.V. Kadavanich, X. Peng and A.P. Alivisatos Appl. Phys. Lett. 69, 1432 (1996).

6. A. Watt, E. Thomsen, P. Meredith and H. Rubinstein-Dunlop. Chem. Commun., 20, 2334 (2004).

7. P.S. Walker, C.E. Finlayson, P.J.A. Sazio, R.J. Curry, D.C. Smith and J. J. Baumberg. J. Mod. Optics. 52 (7), 955 (2005).

8. A.D. Yoffe, Adv. Phys. 42, 216 (1993).

9. A.P. Alivisatos, J. Phys. Chem. 100, 13226 (1996).

10. M. Achermann, M.A. Petruska, S. Kos, D.L. Smith, D.D. Koleski and V.I. Klimov, Nature 429, 642 (2004).

11. D.S. Ginger and N.C. Greenham, Phys. Rev. B 59, 10622 (1999).

12. W.U. Huynh, J.J. Dittmer and A.P. Alivisatos, Science 295, 2425 (2002).

13. V.I. Klimov, A.A. Mikhailovsky, S. Xu, A. Malko, J.A. Hollingsworth, C.A. Leatherdale, H.J. Eisler and M.G. Bawendi, Science 290, 314 (2000).

14. C.E. Finlayson, D.M. Russell, C.M. Ramsdale, D.S. Ginger, C. Silva and N.C. Greenham, Adv. Func. Mat. 12, 537 (2002).

15. M.V. Artemyev and U. Woggon, Appl. Phys. Lett. 76, 1353 (2000).

16. C.E. Finlayson, D.S. Ginger and N.C. Greenham, Appl. Phys. Lett. 77, 2500 (2000).

17. M. Bruchez, M. Moronne, P. Gin, S. Weiss and A.P. Alivisatos, Science 281, 2013 (1998).

18. U. Banin, Y.W. Cao, D. Katz and O. Millo, Nature 400, 542 (1999).

19. N. Tessler, V. Medvedev, M. Kazes, S. Kan and U. Banin. Science, 295, 1506 (2002).

20. J.S. Steckel, S. Coe-Sullivan, V. Bulovic and M.G. Bawendi. Adv. Mater., 15(21), 1862 (2003).

21. R.J. Curry and W.P. Gillin. Curr. Opin. Solid State Mater. Sci., 5, 481 (2001).

22. S. A. McDonald, P. W. Cyr, L. Levina, and E. H. Sargent. Appl. Phys. Lett., 85 (11), 2089 (2004).

23. A. Maria, P. W. Cyr, E. J. D. Klern, L. Levina, and E. H. Sargent. Appl. Phys. Lett., 87 (21), 213112 (2005).

24. A. J. Nozik. Physica E - Low-dimensional Systems and Nanostructures, 14(1-2):115-120, 2002.

25. R.D. Schaller and V.I. Klimov. Phys. Rev .Lett., 92(18):186601, 2004.

26. Randy J. Ellingson, Matthew C. Beard, Justin C. Johnson, Pingrong Yu, Olga I. Micic, Arthur J. Nozik, Andrew Shabaev, and Alexander L. Efros. Nano. Lett., 5(5), 865 -871 (2005). 27. 
